\title{
Simulation and Fabrication of Compounded CMUTs for Medical Application
}

\author{
Yan Li†, Dawei Zhang† and Peiyu Zhang ${ }^{*}$ \\ School of Physics and electronics, Henan University, Henan, China \\ ${ }^{*}$ Corresponding author
}

\begin{abstract}
This paper reports a compounded capacitive micromachined ultrasound transducer (CMUT), which has the potential of CMUTs for multi-frequency transducer applications and medical application both imaging and therapy. The compounded CMUT has a cell with low and high-frequency structures. we introduce a fabrication method involving two silicon-on-insulator (SOI) wafers. The performances of CMUTs are strongly dependent on the geometrical properties of the single cells and their arrangement within each element as well. Our devices are modeled using a finite-element package. Finite element simulations about parametric studies are used for analysis, design, and optimization of CMUT cells to predict device performance. Measured deflections show excellent agreement with modeled performance.
\end{abstract}

Keywords-compounded capacitive micromachined ultrasound transducers; multi-frequency; MEMS; ultrasound; medical application

\section{INTRODUCTION}

There are a number of emerging biomedical applications where it would be advantageous to have an ultrasound transducer that could operate at low frequencies $(1-2 \mathrm{MHz})$ with high power (for example, a treatment mode), yet have the ability to image with high frequencies $(\sim 10 \mathrm{MHz}) \&$ large bandwidth in an imaging mode. Potential applications may include image-guided HIFU-ablation therapy, sensitive contrast agent imaging and radiation-force facilitated targeting, image-guided gene \& drug delivery, and image-guided biomarker release. Both low and high-frequency modes in the same transducer array are desired to ensure accurate coregistration. Unfortunately, such transducers are difficult to fabricate using piezoelectric technology.

Stephens et al [1] demonstrate a custom dual-frequency array transducer which uses a central linear array for highfrequency imaging and two lower-frequency arrays on either side of the imaging array for sonotherapy. Elevational focusing is compromised in both imaging and treatment modes and acoustic impedance matching with two different frequency transducers is non-trivial. Additionally, low- and highfrequency beams do not overlap everywhere as may be desirable. T. Azuma et al. [2] fabricated a $0.5-$ and $2-\mathrm{MHz}$ dual-layer transducer with a frequency-selective isolation layer. Non-trivial acoustic impedance matching was required.

$\dagger$ These authors contributed equally.

Gessner et al [3] demonstrated a dual-frequency (2.5-MHz transmit and $30-\mathrm{MHz}$ receive) transducer for high microbubble-to-background contrast imaging. Their imaging mode relied on highly nonlinear transient responses of contrast agents excited near their resonance frequency and emitting a broadband response received by transducers with frequency response high enough that tissue harmonics are negligible. While excellent images were obtained, unfortunately their system did not use an array and required mechanical scanning.

Capacitive Micromachined Ultrasound Transducers (CMUTs) [4] may offer a viable alternative for dual-frequency array construction due to advances in MEMS microfabrication technologies. A principal difficulty in interlacing high-and low-frequency piezo-electric elements is that different thicknesses of piezoceramics are needed for different frequencies, and fabricating arrays with alternating thickness elements is non-trivial. In addition, acoustic impedance matching layers are required for piezoelectric transducers because of impedance mismatching of piezoceramics to tissue. The requirements of various piezo-electric elements for high or low frequencies different frequencies to matching layers result in the complexities of acoustic impedance matching layers required for piezoelectric transducer more complexity, especially difficult for multi-frequency transducers. Moreover, one consequence of poor impedance matching of piezoceramics to tissue is that heat due to reflected acoustic power can build up and cause transducer damage during highpower operation. In contrast, Compared to pMUTs, although, it is different to accomplishment various thicknesses for cMUTs, the frequency of CMUT cells can be tuned by engineering the width of the membranes. Hence, in the same plane, one may fabricate both large and small membranes.

The dominant advantage is the radiation impedance of CMUTs can be matched to the transmission medium, the matching layer is not required. Because the radiation impedance of CMUTs can be matched to the transmission medium, complexities of acoustic impedance matching layers required for piezoelectric transducers (especially difficult for multi-frequency transducers) are minimized. One consequence of poor impedance matching of piezoceramics to tissue is that heat due to reflected acoustic power can build up and cause transducer damage during high-power operation. CMUT technology has the potential to mitigate such problems.

Our designs are based on the key observation that the acoustic wavelength in water/tissue corresponding to the resonance frequency of CMUT cells is typically significantly larger than the membrane dimensions. This permits low- and high-frequency CMUT elements to be interlaced on a scale smaller than the shortest wavelengths of the array. We 
simulate, fabricate, and test feasibility dual-frequency compounded CMUTs based on these principles.

\section{FinIte-ELEMENT MODELING AND DESIGN CONSIDERATIONS}

Finite element simulations are used for analysis, design, and optimization of CMUT cells to predict device performance. Various membrane geometries, such as hexagonal, circular, square, rectangular, have been used in CMUT transducers. Rectangular elements were chosen in the present study because of the possibility of optimized fill-factor, meaning more transducer surface area per given amount of wafer real-estate.

In order to implement low and high-frequency during application to transducers, membranes with various thickness can be considered to fulfill the requirements. However, it is different to acquire various thicknesses of membrane for different cavities due to the specificity of microfabrication process. Naturally, the membranes and cavies with different dimensions for various resonant frequencies should be taken into account. Based on the consideration above, a novel compounded CMUT architecture with high- and lowfrequency elements is considered.

The cell of compounded CMUT is shown in Figure $1 \& 2$, which has the potential of CMUTs for multi-frequency transducer applications, is proposed and fabricated. The compounded CMUT has a cell with low and high-frequency structures. A compounded cell can be as a low-frequency element or a high-frequency element respectively at the various time periods for different purposes, such as medical image in low-frequency, medical therapy in high-frequency and both in the same time or application for multi-frequency. Moreover, the compounded cells can form combined elements with low and high-frequency elements in composite cells smaller than the shortest wavelength of ultrasound from the array, show in Figure 3. The combined schemes for CMUTs can assure co-registered dual-frequency operation with minimal deleterious grating or sidelobes. Several different schemes of combined CMUTs were offered. Finite element simulations about parametric studies including gap height, membrane thickness, width, and length are used for analysis, design, and optimization of CMUT cells to predict device performance. Our CMUT array has lower predicted sidelobe and grating lobe levels. The fabrication process was shown in Figure 4. Some preliminary experiments are carried out. The experiment results are close to the simulation ones by ANSYS.

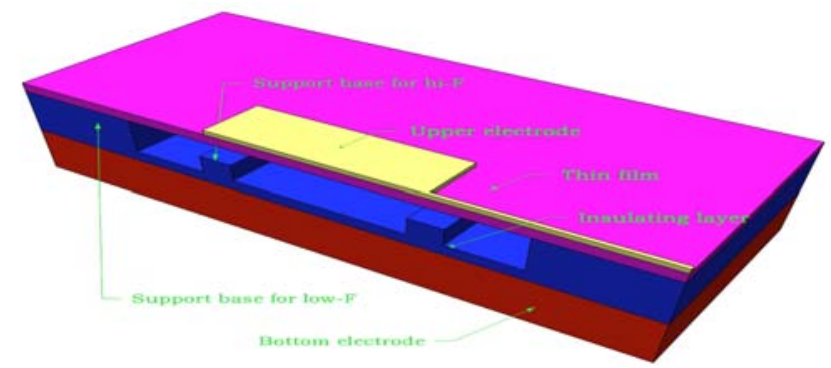

FIGURE I. HALF SECTION VIEW OF A COMPOUND CMUT CELL

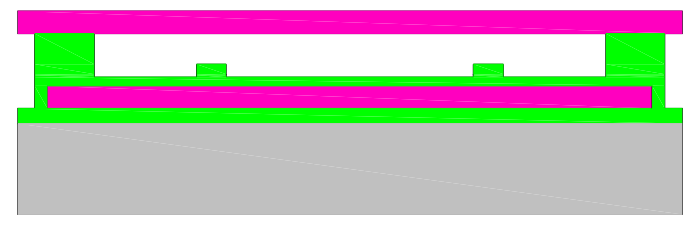

(a)

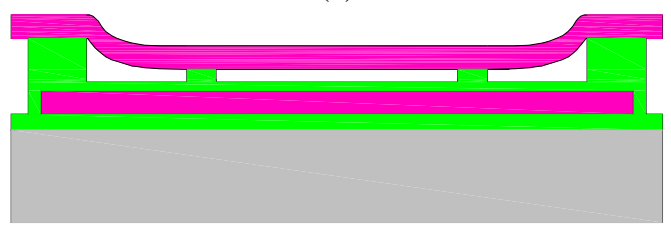

(b)

FIGURE II. VIEW OF SECTION PLANE OF A SINGLE COMPOUNDED CMUT CELL

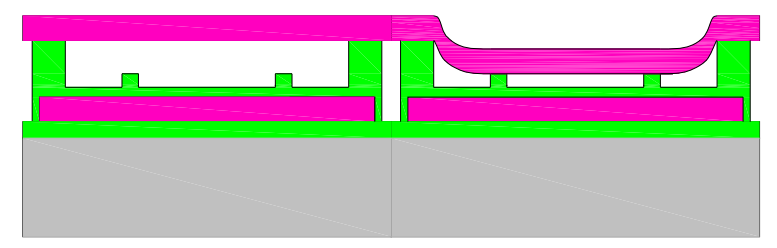

FIGURE III. VIEW OF SECTION PLANE OF A COMBINED CCMUT CELL

\section{FABRICATION}

The compounded CMUTs' fabrication process flow is shown in Figure 4. In the first step of the process, a borondoped SOI wafer is lithographically patterned to form a basic structure including compounded cavities and supporting walls (Figure 4a). Secondly, the wafer is given a thermal growth of oxide (Figure 4b). Next, the $\mathrm{SiO} 2$ layer in the cavities is removed (Figure 4c). After the wafer is given another thermal growth of oxide, the $\mathrm{SiO} 2$ layer on the bottom electrodes are patterned to form the supporting column of the high-frequency cavities (Figure 4d). Then, the third thermal growth of oxide (Figure 4e) is added to form dielectric layer over electrodes.

Another boron-doped SOI wafer is employed in the following steps. The second SOI wafer is patterned as vibrating membranes for different compounded CMUTs' cavities (Figure $4 \mathrm{f}$ ). After that, the SOI wafers is wafer-bonded to the patterned bottom wafer (Figure 4g). After the handle is removed (Figure 4h), the $\mathrm{SiO} 2$ layer is also etched to release the top membrane (Figure 4i). Finally, metallization and patterning is performed to form top electrodes, top electrode bond-pads and the bottom-electrode bond-pads (Figure 4j). 


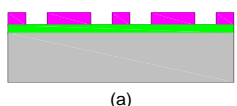

(a)
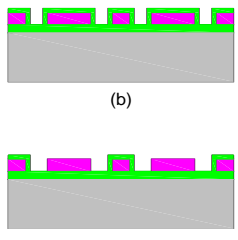

(c)

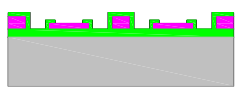

(d)

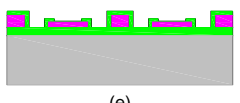

(e)

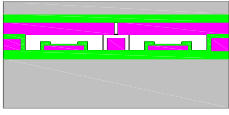

(g)

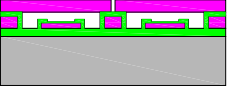

(h)

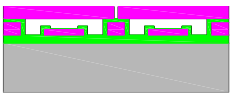

(i)

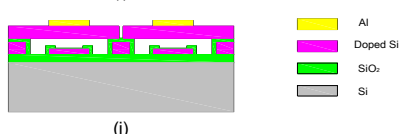

(j)

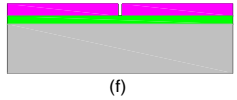

FIGURE IV. FABRICATION PROCESS OF COMPOUNDED CMUTS

\section{SimUlation AND Discussion}

The lateral profiles of simulated and experimental deflection are shown in Figure 5. Figure 5 (a) shows that the simulated lateral deflection profiles at various bias voltages as a function of distance along the membrane width. Comparison of simulated and measured membrane deflections in air with zero applied bias voltage are shown in Figure 5(b). The simulated result is very close to the experimental profile. The discrepancy between the simulation and measurements may be due non-ideal membrane-crimping effects as seen at the edges in Figure 5(b). The relationship between bias voltage and deflection of membrane are depicted in Figure 6, including simulation and experimental results. The simulation and experimental results are very close, which gives us reassurance that the ANSYS models are accurate and reliable.

Comparisons of simulated and experimental CMUT membrane deflection vs. voltage are shown in Figure 6. Bias voltage is increased from 0 to $60 \mathrm{~V}$ and back down to zero for the test. The simulation and experimental results are very close. The measured frequency responses in air for different bias voltages are shown in Figure 7. It is shown that the resonant frequencies shift down as bias voltage increases when using RF Power Amplifier.

\section{CONCLUSION}

A compounded CMUT with high and low-frequency structure are simulated, designed, fabricated, and tested. The simulated and experimental results are very close. Fabricated devices show promising performance as combined imagingtherapy transducers, however, more work is needed to construct and test imaging-therapy linear arrays.
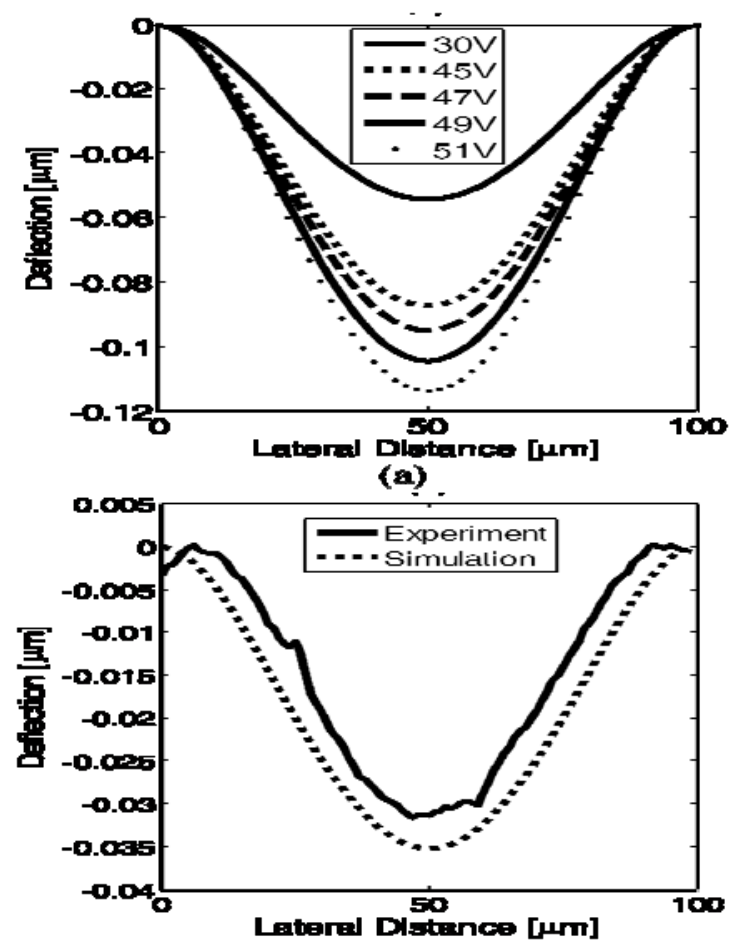

(b)

FIGURE V. COMPARISON OF SIMULATED AND MEASURED MEMBRANE DEFLECTIONS ON THE LATERAL PROFILES

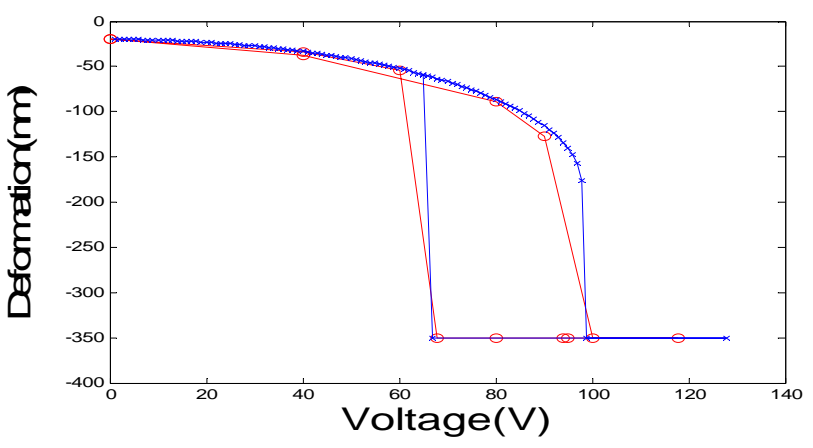

FIGURE VI. THE RELATIONSHIP BETWEEN BIAS VOLTAGE AND DEFLECTION OF MEMBRANE OF CMUTS

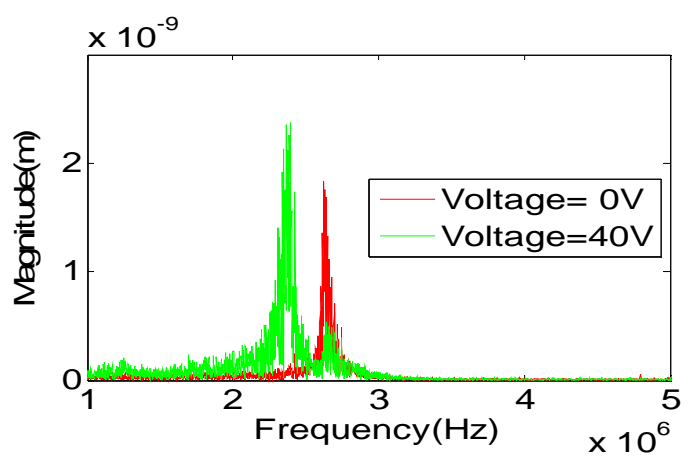

FIGURE VII. VIBROMETER FREQUENCY RESPONSE MEASUREMENTS WITH PSEUDO-RANDOM NOISE INPUT (SHOWN FOR THREE DIFFERENT BIAS VOLTAGES: 0, 40 V) USING A CMUT OF 2 MHZ. 


\section{ACKNOWLEDGMENT}

The authors acknowledge funding support from scientific and technological projects from Office of science and technology of Henan(142102210395), the comprehensive strength project of Midwestern University from Ministry of education of China and the united project for cooperation of enterprise, university and institute from Office of science and technology of Henan.

\section{REFERENCES}

[1] D.N. Stephens, D.E. Kruse, A.S. Ergun, S. Barnes, X.M. Lu, K.W. Ferrara, 2008 "Efficient array design for sonotherapy", Phys Med Biol. 2008 Jul 21;53(14):3943-6

[2] T. Azuma et al., 2010 Dual-frequency ultrasound imaging and therapeutic bilaminar array using frequency selective isolation layer, IEEE UFFC, 57(5), 2010

[3] R. Gessner et al, Blood vessel structural morphology derived from 3D dual-frequency ultrasound images, IEEE IUS 2010

[4] B. T. Khuri-Yakub, Ö. Oralkan, and M. Kupnik, "Next-gen ultrasound," IEEE Spectrum, vol. 46, no. 5, pp. 44-54, May 2009 